\title{
Las condiciones precarias del trabajo de la mujer en México: un estudio de caso en una multinacional en Culiacán, Sinaloa
}

\section{The Precarity Conditions of Women's Work in Mexico: A Case Study in a Multinational in Culiacán, Sinaloa}

\author{
Rosalinda Gámez Gastelum \\ Universidad Autónoma de Sinaloa, México. Doctora en Estudios Organizacionales por la UAM-I; Miembro \\ del SNI, Nivel I. \\ rosalinda.gamez@uas.edu.mx | https://orcid.org/0000-0001-9765-6564
}

\begin{abstract}
Irvin Mikhail Soto Zazueta
Universidad Autónoma de Sinaloa, México. Doctor en Ciencias Económicas-Universidad de Guadalajara. Miembro del SNI, Nivel I. irvin.soto@uas.edu.mx | https://orcid.org/0000-0003-2404-4027
\end{abstract}

\section{Fernanda Barcellos Mathiasi}

\begin{abstract}
Universidad Autónoma de Sinaloa, México. Doctorante en Ciencias Sociales en Doble Titulación con la UAS-Universidad Autónoma de Sinaloa-México y Universidade Federal de Juiz de Fora-MG-Brasil. fernanda.barcellos.faces@uas.edu.mx | https://orcid.org/0000-0002-5354-077
\end{abstract}

Recibido: 25 de julio de 2019. Aprobado: 17 de diciembre de 2019 DOI: $10.25100 /$ lamanzanadeladiscordia.v14i2.8386

Artículo de investigación

Financiación o proveniencia del artículo: Este artículo es parte de un proyecto de investigación en desarrollo sobre las mujeres en el mercado laboral en la ciudad de Culiacán, Sinaloa, por parte de los tres maestros autores de la Universidad Autónoma de Sinaloa.

¿Cómo citar este artículo? / How to quote this article?

Gámez-Gastelum, R., Soto-Zazueta, I. M., y Barcellos Mathiasi, F. (2019). Las condiciones precarias del trabajo de la mujer en México: un estudio de caso en una multinacional en Culiacán, Sinaloa. La Manzana de la Discordia, 14(2), 28-55. doi: 10.25100/lamanzanadeladiscordia.v14i2.8386 


\title{
Resumen
}

El objetivo de este artículo es analizar en qué medida existe precarización del trabajo para las mujeres en el mercado laboral mexicano y cómo estas lo perciben. La investigación utiliza una metodología mixta, con mayor énfasis en el paradigma cualitativo. El artículo parte de la siguiente pregunta: ¿Cuáles son las condiciones laborales de las mujeres mexicanas? Para responder a esta pregunta, el trabajo desarrolla los conceptos de precarización del trabajo, el trabajo decente y la desigualdad de género. En un segundo momento, presenta el estudio de la ENOE en México, como una mirada macrosocial, y finalmente presenta el estudio de caso (microsocial) con trabajadores de un centro de llamadas telefónicas de la empresa multinacional Coppel, ubicada en el noroeste de México. Los hallazgos de la investigación señalan que el trabajo es igualmente precarizado para hombres y mujeres en México. El impacto de este artículo es que cuestiona el mito de que las mujeres son quienes se encuentran en una mayor situación de precariedad laboral en la sociedad posmoderna. Un hallazgo relevante es que encuentra que los jóvenes son un nuevo segmento precarizado en el mundo del trabajo.

Palabras clave: precariedad laboral; desigualdad de género; mercado laboral; trabajo decente; trabajo precario.

\begin{abstract}
The objective of this article is to analyze to what extent there is precarious work for women in the Mexican labor market and how they perceive it. The research uses a mixed methodology. The paper starts from the following question: What are the working conditions of Mexican women? To answer this question, work develops the concepts of job insecurity, decent work and gender inequality. In a second moment, he presents the ENOE study in Mexico, as a macrosocial look and finally presents the case study (microsocial) with workers from a telephone call center of the multinational company Coppel, located in northwestern Mexico. The research findings indicate that the work is equally precarious for men and women in Mexico. The impact of this article is that it questions the myth that women are the ones who are in a greater situation of precarious work in postmodern society. A relevant finding is that he finds that young people are a new precarious segment in the world of work.
\end{abstract} Keywords: Job insecurity; gender inequality; working market: decent job; precarious work. 


\section{Introducción}

Los cambios en el mundo del trabajo contemporáneo que llegaron con la globalización es un tema muy debatido en la academia. Las transformaciones en las condiciones laborales resultado de la flexibilidad, disminución del Estado en la economía, así como las modificaciones en los estatutos jurídicos sobre los derechos del trabajador (Pérez, 2016), han generado un mercado laboral con la característica de inseguridad, que es el eje central de la precarización del trabajo (Standing, 2014a; Kalleberg, 2009).

La precarización del trabajo es el fenómeno posmoderno que reconfigura la categoría social "trabajo", transformándolo en trabajo precario (Antunes, 2000), con bajos sueldos, larga jornada laboral, malas condiciones de salud e higiene laboral, menor representatividad sindical y menos derechos sociales y laborales (Ghai, 2003; Standing, 2014a; Kalleberg, 2009; Antunes, 2009; Beck, 2000). En ese nuevo ambiente laboral también se aparecen las mujeres como un nuevo y representativo actor social, que sufre las condiciones de inseguridad en su vida laboral (Hirata, 2002).

Para enfrentar esos hechos sociales, la OIT propone el concepto de trabajo decente (Ghai, 2003). Este concepto parte de la idea de que el trabajo decente es un eje de identificaciones de las condiciones laborales, en los cuales los países deben de buscar disminuir u obtener, para generar dignidad para la sociedad y sus trabajadores. En el caso mexicano, en los últimos 19 años se han enfrentado cambios importantes en el mundo del trabajo, sobre todo por la incorporación cada vez mayor de la mano de obra femenina. Esto significa que desde 1990, de los 52,9 millones de personas que se han incorporado al mercado de trabajo, más de 13,3 millones son mujeres. De acuerdo con la Encuesta Nacional de Ocupación y Empleo (INEGI, 2005-2018), entre el cuarto trimestre de 2005 y el cuarto trimestre de 2018, cerca de 5 millones de mujeres se incorporaron al mercado laboral.

En este trabajo se parte de la tesis de que existen condiciones diferenciadas de trabajo entre hombres y mujeres mexicanas, ya que no obstante el aumento en la mano de obra femenina, estas enfrentan condiciones laborales que pudieran considerarse precarias. También partimos de la tesis que plantea Hirata (2002), de que en este nuevo ambiente laboral posmoderno son las mujeres, quienes enfrentan de mayor inseguridad laboral.

Este artículo científico busca responder como pregunta principal de investigación: ¿cuáles son las condiciones laborales de las mujeres? y como preguntas de trabajo: ¿en qué medida las mujeres mexicanas son afectadas por la precarización del trabajo?, ¿qué diferencias hay para las mujeres en el mercado laboral de México? y ¿cuál es la percepción de los trabajadores del call center en torno a su trabajo?

La investigación busca responder a estas preguntas por medio de una metodología mixta, con mayor énfasis en el paradigma cualitativo y con el apoyo del análisis descriptivo de la Encuesta Nacional de Ocupación y Empleo de INEGI en el periodo 2005-2018. El artículo es de tipo descriptivo, ya que analiza las condiciones de trabajo de colaboradoras y 
colaboradores (operadores y operadoras telefónicas) del turno matutino del call center del Corporativo "Coppel”, localizado en el edificio La Lonja, en Culiacán, Sinaloa, México, en donde hay 200 trabajadores, de los cuales un 60\% son mujeres, con el propósito de identificar sí tienen condiciones precarias de trabajo y cómo internalizan esta situación en la vida cotidiana de la organización. Esta unidad de análisis fue seleccionada, debido a que es el Centro de Atención Telefónica que cuenta con un mayor número de mujeres.

La metodología usada en este trabajo es mixta, con mayor énfasis en el tipo cualitativo. La metodología (de corte cualitativo), es el estudio de caso simple, el cual permite tener un conocimiento a mayor profundidad de la situación (May, 2004). Esta metodología es flexible y permite explicar en forma detallada el qué de la situación. Las técnicas de recolección de información utilizadas son observación no participante con diario de campo de 30 horas y entrevista semiestructurada a 62 personas que laboran en la organización, de las cuales 60 son operarios y operarias, un jefe de turno y un gerente de zona.

Respecto al paradigma cuantitativo, se utilizó un análisis descriptivo de los datos de la ENOE en el periodo 2005-2018, para revisar las condiciones laborales de hombres y mujeres en México en una mirada macrosocial.

El nivel microsocial se realizó mediante el estudio de caso en un centro de atención telefónica (call center), ubicado en el centro de la ciudad de Culiacán, Sinaloa, México, debido a que en una investigación exploratoria realizada durante los meses de mayo a agosto de 2017 se detecta que en ese lugar existen elementos que pueden configurar las condiciones de un trabajo precario.

Las 62 entrevistas analizadas en esta investigación corresponden a un $50 \%$ de hombres y un $50 \%$ de mujeres, a fin de evitar sesgo en la investigación y lo que se denomina "apropiación de la voz" por parte de las mujeres.

El objetivo de esta investigación es analizar en qué medida existe precarización del trabajo para las mujeres en el mercado laboral mexicano. Más allá de un análisis general de las condiciones laborales, se busca una mirada hacia a la construcción social de los trabajadores sobre género, trabajo precario y decente.

Se parte de la siguiente hipótesis de trabajo: en el call center de Coppel, ubicado en el edificio La Lonja, se labora en condiciones de precarización del empleo, pero los trabajadores la aceptan por ser mujeres, jóvenes sin calificación y madres solteras, por la construcción social que hicieron de sí mismas sobre su origen socioeconómico, los empleos que realizaron previamente, y su condición de desempleados antes de llegar al centro de atención telefónica.

La primera parte del artículo hace un estado del arte del concepto de precarización del trabajo, trabajo decente y precario, en los cuales se comprende como una percepción de inseguridad para los trabajadores. La inseguridad en el aspecto de representatividad laboral, derechos, ingresos, jornada laboral larga, pérdida del empleo, baja calificación, malas condiciones de 
salud e higiene laboral (Kalleberg, 2009). El trabajo decente es el objetivo y plan de trabajo de la OIT (2017) para el mercado laboral mundial, el cual cuenta con condiciones de tiempo, salud, ingresos, representación y sin discriminación para los trabajadores (Ghai, 2003). Al revés, el trabajo precario es la característica del mundo laboral precarizado, inseguro (Standing, 2014a; Kalleberg, 2009; Beck, 2000).

En la segunda parte, partiendo de la tesis que las mujeres son las más afectadas por el trabajo precario, se presenta un apartado teórico sobre la desigualdad de género (Hirata, 2000; Kergoat, 2003; Navarro y Gámez, 2006), que es una construcción social (Ridgeway, 2011). De acuerdo con dicho planteamiento y con la "Teoría de la cola" (Navarro y Gámez, 2006), las mujeres tienen menor oportunidad laboral en competencia con los hombres (Hirata, 2000; Kergoat, 2003).

En un tercer momento se presenta un análisis descriptivo de los datos estadístico de la ENOE, 2005-2018, a partir del cual se presenta una mirada macrosocial y se puede comprender el mercado laboral en México. Bajo un análisis preliminar general descriptivo de los datos de la ENOE 2005-2018, se buscará identificar la cantidad de mujeres en la población mexicana y cuántas tienen trabajos formales con contrato de trabajo (que tienen ingresos, prestaciones, aguinaldo, vacaciones, jornal laboral fija). También se buscará establecer si hay más hombres que mujeres en los trabajos subordinados y remunerados, o sea, formales, con ingresos hasta de 1 salario mínimo, y si hay más mujeres o hombres aun ante la cuestión de la jornada exhaustiva, con más de 48 horas semanales. Con datos generales sobre las variables prestaciones (contratación formal), salario y jornada laboral exhaustiva, se buscará identificar cómo afectan estos a hombres y mujeres. En un primer análisis se alcanza a contestar si las mujeres son objeto de la precariedad laboral más que los hombres.

Después se analiza un estudio de caso en un centro de atención telefónica de una empresa multinacional, Coppel, en Culiacán, Sinaloa, para identificar se existen labores en condiciones precarias y desigualdad de género, en donde los resultados presentan que no existe una diferencia significativa entre el trabajo precario entre hombres y mujeres. Es decir, que el trabajo es precario para ambos géneros y eso cuestiona el mito de que en la sociedad posmoderna son las mujeres quienes enfrentan condiciones laborales más precarias.

\section{¿Qué es la precarización del trabajo?}

La cuestión del trabajo precario como estresante, degradando la individualidad del ser humano no es nuevo en la historia humana. Sin embargo, el objeto de trabajo de esta investigación se centra en el período histórico de la posmodernidad industrial, la llamada contemporaneidad.

El trabajo para el individuo fue tratado en Marx $(1985 ; 2010)$ como una de las maneras del ser humano de diferenciarse del mundo animal, con el fin de adquirir la dignidad humana, la felicidad, siendo un medio para desarrollar el ser social (Hirata, 2002). Es decir, el ser humano se inserta en el mundo a través de lo que es y representa dentro del cuerpo social, dentro de 
la convivencia con el otro y el status quo que recibe por las funciones que realiza (Hirata, 2002).

Pero si por un lado tenemos el trabajo como agente del desarrollo individual, propulsor de la dignidad humana (Marx, 2010), de la realización de los proyectos individuales y de perspectivas de futuro. Por otro lado, tenemos que la precarización del trabajo en el capitalismo moderno está cambiando la actividad laboral (Antunes, 2000), ya que se transformó en mercancía de cambio, alienado, fetichizado (Marx, 1985). Si antes el trabajo era visto como finalidad central del ser social, ahora se transforma en un medio de subsistencia, con el objetivo de valorizar el capital (Antunes y Braga, 2009).

En ese sentido (Antunes, 2009) plantea que la modernidad y posmodernidad no tendrían fuertes efectos sociales en el mundo del trabajo, debido a la tecnología. Sin embargo, en un trabajo posterior reconoce que lo que sí existen cambios en el mercado laboral, con la introducción del desempleo estructural, el outsourcing, el trabajo temporal o parcial y los salarios bajos (Antunes, 2015). Es decir, que los efectos de la posmodernidad están generando las características del trabajo precario y aumentando la concentración de riqueza (OIT, 2017).

El término "trabajo precario", entre otros sinónimos que se han utilizado para describir las transformaciones que el "mundo del trabajo", ha estado sufriendo cambios durante toda la legislación mundial, en América Latina, y por supuesto, México, con una retirada de las políticas de Estado y de avance y agendas de mercado (Standing, 2014a). El trabajo precario empieza a ser una preocupación de la década de 1970 (Antunes, 2009; Kalleberg, 2009; Santos, 1993; Sassen, 2010). Como plantea Kalleberg (2009), trabajo precario es aquel "incierto, impredecible, y en el que los riesgos son asumidos principalmente por el empleado, y no por los empleadores o el gobierno" (p. 21). Este autor plantea que el aumento del trabajo precario en el mundo es el resultado de la globalización, la interdependencia económica y la expansión del neoliberalismo. En América Latina, el trabajo precario surgió con el aumento de la privatización, la desregulación del mercado y el avance del neoliberalismo en la década de 1990 (Beck, 2000; Kalleberg, 2009).

La globalización es un hecho y sus efectos tienen implicaciones en la sociedad contemporánea, y, sobre todo, con relación a hombres y mujeres, afectan desigualmente el mercado laboral (Hirata, 2002). La paradoja de la globalización es que, según investigaciones económicas y sociales, la mujer participa más del mercado laboral; es decir, tiene mejor acceso a un empleo, pero las condiciones de trabajo que le son ofertadas son las más precarias con relación a salarios, jornada laboral, contrato de trabajo, prestaciones y condiciones de salud (Abramo, 2006; Hirata, 2002). Al respecto, Bauman (2010), teórico posmoderno, en su trabajo la "Modernidad líquida", identifica que uno de los elementos de la globalización es generar la inseguridad en el mundo, porque si antes, en la Modernidad tendríamos al Estado como el actor social capaz de contestar y realizar los deseos y agendas políticas de la sociedad, en la sociedad Posmoderna existen muchas instituciones internacionales y actores globales que pulverizan el poder y la capacidad de contestar esas demandas. Según el autor, en esa 
categoría existe una ética del trabajo, sobre todo, después de las ideologías y religiones protestantes (Bauman, 2000). El objetivo fue formar un ethos $^{1}$, una nueva forma de mirar la vida basada en el trabajo, en el lucro, en el suceso por el dinero y en el reconocimiento social por la profesión y dinero, impuesta en una fuerte disciplina (Bauman, 2000).

Al revés, en el "mundo líquido" de la Postmodernidad, el trabajo' es caracterizado con el atributo de la inseguridad (Beck, 2000); o sea, aunque se capacite y sea un "buen" trabajador, no hay certeza sobre los sueldos, las jornadas laborales, si los derechos sociales y de la seguridad serán cumplidos, o si habrá trabajo y puesto de empleo (Pérez, 2016).

En el libro "Estado de crisis", Bauman y Bordoni (2016) sostienen que, además de la inseguridad, aparece la "financeirización" de la economía, la cual es otro factor relevante al trabajo y a las condiciones laborales que cambiaron con la globalización. O sea, en palabras marxistas, anteriormente el capitalista estaba preocupado por el lucro y después de obtenerlo iba a reinvertirlo otra vez en su empresa, tenido la posibilidad de generar más empleo, mejores sueldos y abonos a los operarios. Pero ahora, con una nueva visión empresarial, el lucro se va a bancos de investimento, generando concentración de la riqueza y no distribución (Bauman y Bordoni, 2016). Es así como estamos en una fase social de mucha fuerza del capital financiero frente a los trabajadores, que no tienen la fuerza del dinero, ni del Estado, y tampoco creen en instituciones estatales y sociales como los sindicatos (Bauman, 2010).

El trabajo, sobre todo, el trabajo de la mujer está cada vez más vulnerable, pues ha sido muy flexibilizado, sin contratación formal o disminución de derechos sociales y laborales, dependiente del mercado mundial en una economía cada vez más globalizada (Hirata, 2002). La mencionada globalización viene junto con la migración y sus impactos en el mercado laboral (Sassen, 2010). Sobre estos temas, el economista Guy Standing (2014a, 2014b) teoriza sobre la precarización del trabajo y sostiene que el "precariado" es una nueva clase social que surgió con la globalización (Valencia, 2015). Esta clase se caracteriza por no contar con condiciones dignas de trabajo, desarrollando trabajo precario; o sea, que no posee seguridad de tener el empleo, un sueldo, los derechos sociales cumplidos, ni derechos garantizados o fiscalizados por el Estado, pues son migrantes (Standing, 2014a). Esta clase social formada por trabajadores migrantes precarizados son una buena opción para los empresarios que desean disminuir el costo de la producción, pero es un factor generador de desigualdades y de incumplimiento del Estado de Bienestar Social (Stiglitz, 2002).

Ulrich Beck (2000) propone un nuevo concepto sobre la precariedad del trabajo con el nombre de "brasileñización" del mercado laboral. Es decir, que el mercado laboral está cada vezmás

\footnotetext{
${ }^{1}$ (...) la apelación de la ética del trabajo ocultaba el primitivo impulso de apartar a los obreros de la racionalidad del mercado, que parecía ejercer un efecto nocivo sobre la dedicación de sus tareas. Bajo de la ética del trabajo se promovía una ética de la disciplina, ya no importaban el orgullo o el honor, el sentido o la finalidad. El obrero debía trabajar con todas sus fuerzas, día tras día y hora tras hora, aunque no viera el motivo de ese esfuerzo o fuera incapaz de vislumbrar su sentido último (Bauman, 2000, p. 20)
} 
inseguro, sin garantías laborales, sin derechos cumplidos, con bajos sueldos, pocas condiciones de salud, muchas horas laborales y desarrollo de muchas funciones. Esas características, afirma Beck (2000), son cada vez más comunes en los trabajos de todo el mundo y sobre todo en Europa, donde realiza su análisis espacial de investigación.

El precariado entonces es una fracción social que se diferencia del proletariado pues ese tiene derechos garantizados por leyes y sindicatos que los representan (Braga, 2012; Valencia, 1999 y 2015), seguridad y beneficios sociales. Los primeros no son reconocidos por los trabajos o funciones que ejercen, no trabajan en la función que están capacitados, es una clase políticamente conservadora, no tiene sindicatos que los representa, ni tampoco derechos que les garantizan empleo, despido arbitrario, no tienen horas fijas de trabajo, ni sueldo garantizado, y siguen sin identidad ocupacional (Standing, 2014b). Por eso, Standing (2014a) identifica el trabajador precario con estas siete características: inseguridad en mercado laboral; inseguridad en el empleo; inseguridad en el puesto de trabajo; inseguridad en el propio trabajo; inseguridad en la reproducción de las habilidades; inseguridad en los ingresos; inseguridad en la representación.

Entonces, el autor reconoce el precariado como producto del desarrollo económico contemporáneo, de tal manera que se contribuye a la formación de una nueva clase social. En esa misma línea argumentativa Standing (2014a), apunta seis hechos sociales: flexibilización del trabajo; no hay identidad ocupacional; no pueden realizarse en una carrera profesional; protección laboral insuficiente; derechos sociales y laborales siempre reduciendo o sobre ataques; migración.

Por su parte, el sociólogo Arne Kalleberg (2009), quien desarrolla el tema de la precariedad laboral con base en el manifiesto de la Organización Internacional del Trabajo (OIT), distingue siete puntos de calidades de la precariedad del trabajo; es decir, plantea que existen rasgos que caracterizan a una obra tan precaria. Es así como el autor identifica la similitud entre la precariedad y la inseguridad, como sinónimos. A continuación, se presentan las siete características:

1) la incertidumbre en el mercado de trabajo (falta de oportunidades de empleo);

2) la incertidumbre del trabajo (protección inadecuada contra la pérdida de empleo o despido arbitrario);

3) la inseguridad laboral (incapacidad para continuar en una ocupación en particular debido a la falta de límites de artesanía y calificaciones de trabajo);

4) la inseguridad seguridad y salud (malas condiciones de seguridad y salud ocupacional);

5) experiencia de reproducción de incertidumbre (falta de acceso a la educación básica y la formación profesional);

6) la inseguridad de ingresos (ingresos insuficientes; sin pago garantizado o la expectativa de un nivel adecuado de ingresos actuales y futuros); 
7) la representación de la inseguridad (falta de derechos individuales en las leyes y los derechos colectivos para negociar) (Kalleberg, 2009, p. 25).

Paralelo a los conceptos de precariedad laboral en el mercado de trabajo y trabajo precario, existe el concepto de trabajo decente (Gálvez, Gutiérrez y Picasso, 2011), que es el más utilizado por la Organización Internacional de Trabajo (OIT), como paradigma a ser buscado ampliamente por todas las sociedades para el trabajo (Ghai, 2003). Este concepto es suficiente para definir condiciones mínimas o más sencillas que deben tener todos los trabajadores, incluso los informales, autónomos, asalariados y temporales.

Para el sociólogo Minor Mora (2012), la precariedad laboral ${ }^{2}$ que existe dentro de esta formación contemporánea del Estado tiene al empleo precario como objeto y sus variables serían los trabajadores: 1) sin contrato formal de trabajo; 2) sin prestaciones (aguinaldo y vacaciones); 3) salario inferior a 2 salario mínimos; 4) sin seguro social; 5) desregulación de la jornada laboral (más de 48 horas semanales o menor que 35 horas semanales).

Para Anker, Chernyshev, Egger, Mehran y Ritter (2003), el trabajo decente puede ser expuesto de manera más clara en los seis puntos: a) oportunidades de trabajo; b) trabajo en condiciones de libertad; c) trabajo productivo; d) equidad en el trabajo; e) seguridad laboral; y f) dignidad laboral. Pero como se puede observar, son hechos sociales muy amplios y de difícil medición. Por eso, Anker et al. (2003) establecen una medición del concepto del trabajo decente con once categorías de análisis:

1. oportunidades de empleo;

2. trabajo inadmisible (da la idea de trabajo en condiciones de libertad);

3. remuneración suficiente y trabajo productivo (da la idea de trabajo productivo);

4. trato justo en el trabajo;

5. conciliación del trabajo con la vida familiar;

6. diálogo social y relaciones laborales;

7. dan la idea de equidad y dignidad en el trabajo;

8. seguridad en el trabajo;

9. protección social, estabilidad y seguridad del empleo.

\footnotetext{
2 "Para efectos de medición se han considerado las cuatro dimensiones principales del concepto de precariedad laboral antes señaladas: inseguridad sobre la continuidad de la relación laboral, degradación y vulnerabilidad de la situación del trabajo, incertidumbre e insuficiencia de los ingresos salariales y desprotección social del trabajador. Se ha introducido un pequeño matiz, pues se ha agregado una quinta dimensión que aludió a la desregulación de la jornada laboral para intentar captar un factor temporal en materia de precarización laboral resultado del cambio en las estrategias de gestión del tiempo de trabajo inducidas por la flexibilidad laboral" (Mora, 2012, p. 96).
} 
Queda claro que los conceptos de "precaridad" laboral, "precarización" del trabajo y "trabajo precario" no son lo mismo. Cabe destacar que precariedad laboral son factores externos de la sociedad y economía, que proporcionan ciertos atributos y características hacia al trabajo contemporáneo (Kalleberg, 2009; Standing, 2014a). En tanto, la precarización laboral es el fenómeno social de empeoramiento de las condiciones del trabajo, el cual es un proceso a largo plazo y no es algo fijo (Beck, 2000; Sassen, 2010). A su vez, de acuerdo con Mora (2012), el "trabajo precario” es otra categoría de análisis sobre la calidad del trabajo, calificación y atributo que especifica un tipo de trabajo.

Así que después de presentar los conceptos sobre la precariedad del trabajo, trabajo precario y trabajo decente, esa investigación se va a detener en la teoría de desigualdad entre hombres y mujeres, la desigualdad de género, para después analizar los datos y obtener respuestas sobre si existe o no desigualdad entre hombres y mujeres en la sociedad mexicana.

\section{Trabajo de la mujer y la desigualdad de género}

Los cambios en el mundo laboral han sido cada vez más fuertes y han presentado atributos diferentes con el pasar de los años, sobre todo en aspectos como el trabajo de la mujer o la cuestión de género en el mercado laboral (Ridgeway, 1992; Navarro y Gámez, 2006). La Organización Internacional de Trabajo (OIT) presenta diversos informes sobre temáticas laborales en el mundo, y la cuestión de género ha sido siempre constante, pues los datos muestran que cada vez existen más mujeres en el mercado laboral, pero sus ingresos, jornadas laborales, prestaciones, trabajo formal, prestaciones, pensiones y condiciones de salud laboral, son siempre peores para las mujeres que para los hombres (Ridgeway, 1992; Sen, 2000; Ghai, 2003; Abramo, 2006).

La desigualdad de género se manifiesta en varios países del mundo en el sentido de renta, es decir, el desnivel salarial entre hombres y mujeres en el mercado de trabajo (Abramo, 2006). Aunque ha habido una inserción masiva de las mujeres en el mercado de trabajo en la sociedad contemporánea, principalmente en la post Segunda Guerra Mundial, aún persisten desigualdades en atribuciones sociales, especialmente en tareas domésticas (Ridgeway, 2011).

Sobre la cuestión de la desigualdad de género, hay una acumulación de desigualdad que se manifiesta a través del aspecto de la desigualdad de estatus (Ridgeway, 2011). O sea, el estatus de género que dan a la desigualdad entre los individuos es organizada con base en la clasificación social (Tilly y Tilly, 1998), como machos o hembras, en lugar de los atributos o funciones desempeñadas (Navarro y Gámez, 2006). Sin embargo, las creencias de estatus de género no "actúan" solas, ellas son dependientes de la desigualdad posicional que crean diferencias de recursos y de energía entre hombres y mujeres (Ridgeway, 2011). 
Según Cecilia Ridgeway (2014), que trae para el estudio de las desigualdades el objeto "estatus", argumenta que este no es secundario para la comprensión de las relaciones sociales que generan la desigualdad, pues es un mecanismo independiente y relevante para estudiar. O sea, la profesión escogida, así como el papel social que el individuo desempeña está atrapado mucho más al "estatus" social que se desea obtener con la función desempeñada, que con el salario o dinero obtenido (Ridgeway, 2014):

De esta forma los individuos forman creencias de estatus que el "tipo" de persona que tienen más recursos (por ejemplo, los blancos) son "mejores" que los "tipos" con menos recursos. (...) las creencias de estatus resultantes son compartidas por dominantes y subdominantes igualmente, legitimando la desigualdad (p. 3).

Así, la manera de fortalecer la creencia del estatus social, de acuerdo con Ridgeway (2014), es fortalecer el "estilo de vida", caracterizado por "palabras", "gustos" estéticos, hábitos, lugares a ser frecuentados, entre otros ethos que caracterizan a las clases sociales y las diferencias. En una sociedad meritocrática, el estatus es legitimado (Ridgeway, 2014).

Para comprender el género, es importante reconocer su característica no exclusiva biológica, y abordarlo como una construcción social de un fenómeno cultural que se va a reflejar en la subjetividad del actor social, su identidad, interpretación del mundo, experiencias, instituciones y estructuras (Navarro y Gámez, 2006). Así, lo que determina las expectativas y comportamientos de género no es el sexo biológico sino el haber vivido desde el nacimiento una serie de experiencias, ritos, valores y costumbres atribuidos (Navarro y Gámez, 2006, p. 21).

El género, por tanto, puede ser usado como una credencial. Esta imagen social del individuo se construye con base en los padrones e inmediatamente se expone en una presentación, en un encuentro, antes de cualquier conocimiento individual (Hirata, 2000; Kergoat, 2003). Pero existen suposiciones sobre la desigualdad que son incorporadas por significados y creencias sociales (Ridgeway, 2011) y discriminaciones (Navarro y Gámez, 2006).

Esta teoría es unísona con la "teoría de la cola" que plantea que, en el mercado laboral, para asumir un puesto de trabajo hay una "cola" en la cual están primeros los hombres, blancos y luego negros, seguido de las mujeres, blancas y negras (Navarro y Gámez, 2006); o sea, la mujer sufre un cúmulo de desigualdad en el mercado laboral (Ridgeway, 2011). Por tanto, la cuestión de la desigualdad de género en el mercado laboral es, sobre todo una cuestión del estatus social (Ridgeway, 2014), o mejor dicho, la construcción social de lo que es ser hombre y de lo que es ser mujer en la sociedad (Hirata, 2000; Ridgeway, 2011, 2014; Kergoat, 2003). 
Lo que se sostiene en ese trabajo es que además de la cuestión biológica (Navarro y Gámez, 2006), existen construcciones sociales que desde del nacimiento tienen preestablecidos actos, juegos, trabajos, manifestaciones "de hombres" y de "mujeres". Por ejemplo, los juegos que estimulan la creatividad, la habilidad de fuerza física y manual son enseñadas a los niños, y para las mujeres se les atribuye aquellos relacionados con las actividades del hogar, los juegos como ama de casa, madre, lo cual desde temprano desarrolla ciertas habilidades y miradas hacia el mundo (Hirata, 2000; Kergoat, 2003).

Esta construcción social del estatus de "ser mujer" genera discriminación ${ }^{3}$ social (Horbath y Gracia, 2014), hecho que la Organización Internacional de Trabajo (OIT) busca erradicar, pues dicha desigualdad de género (Ghai, 2003) impacta las tasas económicas de diferencia salarial, derechos sociales, jornada laboral, prestaciones, posibilidad de empleo formalizado por contrato de trabajo, impide el alcance del trabajo decente (Abramo, 2006), no precario, generando una sociedad con características de la precarización laboral (Bauman, 2000; Beck, 2000, Standing, 2014a; Kalleberg, 2015).

Es por ello que la OIT promueve el trabajo decente (Ghai, 2003) como una agenda de promoción para el mundo laboral, y uno de los objetivos para alcanzar este tipo de trabajo es la búsqueda por la no discriminación (Horbath y Gracia, 2014), y la equidad en el mercado laboral; es decir, que las mujeres tengan derechos y realidades semejantes a los hombres, mismos ingresos para las mismas funciones, oportunidad de estudio y crecimiento profesional, horas de trabajo ecuánime, condiciones de salud e higiene laboral, prestaciones y contrato laboral dignos. Al respecto, Abramo (2006) plantea:

Trabajo decente significa un trabajo productivo y remunerado para hombres y mujeres. Para las mujeres eso supone, al mismo tiempo, a) aumentar sus posibilidades de acceso al trabajo remunerado y oportunidades de generación de ingreso; b) disminuir la invisibilidad del trabajo realizado en diversas esferas no reconocidas en tanto tal; c) mejorar la calidad de sus empleos (p. 16).

Recientemente, en 2017 fue divulgado el Informe Global sobre Salarios por la OIT que afirma que desde 2012 los crecimientos de los salarios reales a nivel global, a excepción de China

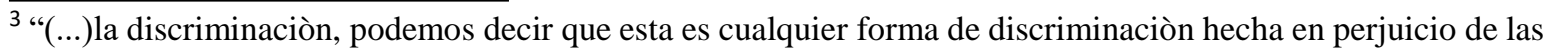
mujeres en general, o de un grupo determinado, por el simple hecho de pertenecer al género femenino. (...) Es de considerarse tambièn dicho fenómeno en el seno familiar visible, tales como la falta de facilidades para el cuidado infantil, la carencia de modelos de referencia para el rol femenino y de asesorìas para la mujer; las barreras invisibles son, por ejemplo, las actitudes de prejuicio, las creencias y la conducta exclusivista definida por los hombres; el impacto psicològico inconsciente de las organizaciones patriarcales sobre la motivaciòn de la mujer, su autoestima y la relaciòn reflexiva entre el contexto biogràfico y el conocimiento. Esto es a veces desconocido aun para las mismas mujeres, ya que el impacto patriarcal se filtra hasta en sus razonamientos para hacer una autoevaluaciòn reflexiva (Navarro y Gámez, 2006, p. 23).
} 
que tuvo un aumento, tuvieron una disminución porcentual de 1,6\% en 2012 para 0,9\% en 2015 del valor (OIT, 2017):

En 2015 los salarios reales cayeron cerca de 1,3 por ciento en América Latina y el Caribe (sobre todo debido a la baja de los salarios en Brasil), y 5,2 por ciento en Europa del Este (debido a la baja de los salarios en la Federación Rusa y Ucrania) (OIT, 2017, p. 1, traducción libre).

Pero en países desarrollados, como aquellos pertenecientes al G20, por ejemplo, los salarios tuvieron un aumento, pasaron de 0,2\% en 2012 a 1,7\% en 2015, la tasa más elevada de los últimos 10 años. En el año de 2015 en Estados Unidos el crecimiento real de los salarios tuvo un aumento de 2,1\%; 1,5\% en Europa del Norte, y 1,9\% en los países de la Unión Europea. Otro dato interesante del informe de salarios de la OIT (2017) es acerca de la diferencia salarial entre hombres y mujeres que se acentúa con la mejor calificación y los mejores puestos de trabajo; es decir, cuanto más elevado el estatus profesional, las funciones, más topo de la pirámide del empleo, más hay diferencia salarial existe entre hombres y mujeres (OIT, 2017).

Es así como el informe concluye que el salario es fundamental para una integración económica y social (OIT, 2017). El estancamiento de los salarios por la cuestión social, la diferenciación entre el crecimiento económico y el no crecimiento de los salarios, implica una insatisfacción de la sociedad, pues el trabajador no percibe mejoras en su vida y su familia (OIT, 2017). Además de eso, en la cuestión económica, el hecho que no aumenten los salarios implica que los trabajadores van a consumir menos y disminuye el crecimiento económico (OIT, 2017).

En México, los estudios indican que existe desigualdad salarial entre hombres y mujeres, aunque desempeñen la misma actividad laboral (Navarro y Gámez, 2006; Abramo, 2006). Concluye las expertas Gloria Navarro y Rosalinda Gámez (2006), que eso se da en el caso mexicano por la relación fuerte que existe entre la familia y sus principios: a) la supremacía indiscutible del padre y; b) el necesario y absoluto auto sacrificio de la madre (Navarro y Gámez, 2006, p. 27).

Entonces para un desarrollo económico sostenible, que pueda generar disminución de la desigualdad social y discriminación, es importante generar políticas públicas que además de integrar la mujer en el mercado laboral, proporcionen un trabajo decente (Gálvez, Gutiérrez y Picasso, 2011), seguro, sin los efectos de la precariedad laboral, con ingresos, prestaciones y jornada laboral iguales a los hombres, así como ofertando iguales oportunidades laborales (García y Pérez, 2007). 
Es importante, además, la cuestión de proporcionar debates y reconocimiento social de la desigualdad y discriminación, para que las próximas generaciones también empiecen a tener/crear una construcción social de género diferente de la que existe, desde niños/niñas, para que sean capaces de no vincular capacitación y actividades para más de las cuestiones biológicas y de esta manera, haciendo una transformación social por la modificación a la mirada cultural.

\section{Las mujeres en el mercado laboral mexicano: análisis descriptivo de la ENOE 2005- 2018}

Durante los últimos 19 años, uno de los cambios más importantes que ha experimentado el mercado de trabajo mexicano ha sido la incorporación de las mujeres a la actividad remunerada. La inserción laboral de las mujeres en los últimos años en México presenta aspectos innegablemente positivos. No solo ha aumentado el número de mujeres que quieren trabajar en relación con el total de la población femenina, sino que, en efecto, hay más mujeres empleadas, tanto asalariadas como empleadoras y autónomas, y las más jóvenes se incorporan al mercado de trabajo con un mayor nivel formativo que las generaciones anteriores.

En concreto, en México, desde 1990, de los 52,9 millones de personas que se han incorporado al mercado de trabajo, más de 13,3 millones han sido mujeres. De acuerdo con la Encuesta Nacional de Ocupación y Empleo - ENOE (INEGI, 2005-2018), entre el cuarto trimestre de 2005 y el cuarto trimestre de 2018, cerca de 5 millones de mujeres se incorporaron al mercado laboral.

La población total mexicana es formada por 125.191 .900 de personas, de los cuales 48,24\% son hombres y 51,76\% son mujeres. En el censo del año 2000 eran 97,4 millones de mexicanos, de los cuales el 48,6\% eran hombres y 51,4\% eran mujeres (Navarro y Gámez, 2006). Aún más, la ENOE (INEGI, 2005-2018) muestra que el crecimiento acumulado de la población ocupada diferenciada por género, entre el cuarto trimestre de 2005 y el cuarto trimestre de 2018, fue de $23 \%$ para los hombres y $32 \%$ para las mujeres. Un análisis desagregado revela que la direccionalidad y magnitud del crecimiento en el empleo por género es bastante diferente entre estados.

En este sentido, las mejores ciudades de México para el trabajo de los hombres son Cancún, Saltillo y Querétaro (Guzmán, 2009). Por su parte, para el desarrollo profesional de las mujeres, Ciudad de México y Aguascalientes presentaron mejores datos (Guzmán, 2009).

Según cifras de la ENOE (2005-2018), al cuarto trimestre de 2018 casi la mitad de los trabajadores con contratos de trabajo (Trabajadores subordinados y remunerados, según 
clasificación de la ENOE) —indistintamente de su género- recibía ingresos mensuales que no superan los dos salarios mínimos. Por lo que se infiere que más allá de una cuestión de género, el trabajo en México se concentra en los puestos menos remunerados.

Pero dentro del eje de trabajadores subordinados que ganan hasta un salario mínimo, 47,87\% son hombres y $52,13 \%$ son mujeres, o sea, las mujeres se ven más afectadas por los más bajos ingresos.

Los datos más recientes del último semestre de 2018 apuntan que, entre los trabajadores con contrato de trabajo formal, $61,68 \%$ son hombres y $38,32 \%$ son mujeres. Ese eje ya se puede observar un elemento de desigualdad de género en la sociedad mexicana, pues la mayor parte de la población activa son mujeres, pero el mercado laboral formalizado, trabajo subordinado y remunerado, la mayoría son hombres.

En el análisis diferenciado entre hombres y mujeres, muestra que ellas tienden a recibir menos ingresos que ellos (ENOE: INEGI, 2005-2018). Por cada mujer que accede a los trabajos mejor pagados (mayor a 5 salarios mínimos) habrá 3 hombres que ganen lo mismo. En el extremo opuesto, de cada 100 trabajadores en el rango de ingresos más bajo (hasta 1 salario mínimo), habrá 4 mujeres más que hombres (ENOE: INEGI, 2005-2018).

Para lograr que más mujeres alcancen los ingresos más altos, expertos apuntan que uno de los mayores retos es incrementar la disponibilidad para trabajar. Sobre las horas laboradas y jornada laboral extensiva la ENOE apunta que uno de cada tres hombres dice trabajar más de 48 horas semanales, frente a una de cada cinco mujeres.

En México, el trabajo femenino es categóricamente distinto al trabajo masculino en términos de crecimiento, dispersión salarial y concentración sectorial, pero ¿sucede lo mismo con las cifras de desocupación? De acuerdo con el IMEGI, poco más de 1,8 millones de personas que buscan activamente trabajo no lo encuentran, lo que implica que la tasa de desempleo es igual a 3,3\%. La desocupación para hombres y mujeres es de $59 \%$ y 41\%, respectivamente. Así que, en el caso de población desocupada en México, la hipótesis de investigación no se comprueba, pues hay un porcentaje importante mayor de hombres precarizados frente a las mujeres.

Bajo un análisis preliminar general descriptivo de los datos de la ENOE 2005-2018, se puede concluir que las mujeres son la mayor parte de la población mexicana, pero los trabajos formales, con contrato de trabajo (que tienen ingresos, prestaciones, aguinaldo, vacaciones, jornal laboral fija) son más ocupados por hombres que mujeres. Los puestos de trabajo subordinado y remunerado, o sea, formales, con ingresos hasta un1 salario mínimo, también 
hay más mujeres que hombres. Sobre la jornada laboral exhaustiva, con más de 48 horas semanales, la mujer es la más afectada por esa característica.

La conclusión de los datos generales, en una mirada previa, es que existe más precariedad laboral para las mujeres en el mercado laboral mexicano, pues son afectadas por jornadas laborales más largas, ingresos más bajos y menos condiciones de un contrato de trabajo formal, aunque sean la mayor parte de la población de México.

\section{Aspectos metodológicos}

Este artículo científico busca responder a la pregunta principal de investigación ¿cuáles son las condiciones laborales de las mujeres en México? y a las preguntas de trabajo:

¿En qué medida las mujeres mexicanas son las más afectadas por la precarización del trabajo? ¿Qué diferencias hay para las mujeres en el mercado laboral de México? ¿Cuál es la percepción de los trabajadores sobre su empleo en el call center?

El trabajo utiliza metodología de la investigación mixta, pero con un mayor énfasis en el paradigma cualitativo. Se auxilia del cuantitativo para el nivel macrosocial a través del análisis descriptivo de la Encuesta de Ocupación y Empleo (ENOE) en el periodo 2005-2018, lo cual permite identificar cuál ha sido la evolución del trabajo en el ámbito mexicano en la denominada etapa posmoderna.

En el apartado de metodología cualitativa, el alcance de la investigación es de tipo descriptivo, ya que revisa las condiciones de trabajo de colaboradoras y colaboradores (operadoras y operadores telefónicos) del turno matutino del Antúnez, Ricardo (2000). call center del Corporativo “Coppel”, localizado en el edificio La Lonja, en el centro de Culiacán, Sinaloa, México, en donde laboran 200 empleados, de los cuales un 60\% son mujeres. Esta revisión tiene el propósito de identificar sí presentan condiciones precarias de trabajo y cómo interiorizan esta situación en la vida cotidiana de la organización.

La metodología usada es el estudio de caso simple, el cual permite tener un conocimiento a mayor profundidad de la situación (May, 2004). Las técnicas de recolección de información utilizadas fueron observación no participante con diario de campo de 30 horas y entrevista semiestructurada a 62 personas que laboran en la organización, de las cuales 60 son operarias, 1 jefe de turno y 1 gerente de zona. Del total de la muestra de 62 entrevistas realizadas a personal operario del centro de atención telefónica, el $50 \%$ fueron hombres y el $50 \%$ fueron mujeres, a fin de evitar sesgo en la investigación y evitar la apropiación de la voz del sexo femenino, lo cual podría afectar la objetividad del trabajo de campo. 
Esa muestra representa el $31 \%$ del total de los empleados y fue a conveniencia, debido a que se buscó trabajar el 50\% de la muestra (100 empleados), pero los directivos al enterarse del trabajo de campo, prohibieron al personal responder a las entrevistas. Esa falencia trató de ser subsanada entrevistando adicionalmente, a cinco exoperadores telefónicos (que tenían menos de tres meses de haber salido de la empresa).

De la muestra analizada, se destaca que el $80 \%$ son estudiantes universitarios, $10 \%$ estudian la preparatoria y el resto tiene escolaridad secundaria. También se resalta que del $50 \%$ de la muestra de mujeres, un $30 \%$ son jefas de familia y de estas un $20 \%$ son madres solteras con más de un hijo en edad infantil.

Respecto a la unidad de análisis, se seleccionó el Centro de Atención Telefónica, que funciona en el tercer piso del edificio "La Lonja" por tener un mayor número de mujeres y de mujeres jefas de familia. Trabajan en un horario de 6:30am a 1:40pm, con un receso para comer de media hora de 8:00 a 8:30am.

El nivel microsocial se realizó mediante el estudio de caso en un centro de atención telefónica (call center), el cual se seleccionó después de una investigación exploratoria realizada durante los meses de mayo a agosto de 2017 con trabajadoras del edificio "La Lonja", quienes son estudiantes universitarios en el turno nocturno y se detecta que en ese lugar existen elementos que pueden configurar las condiciones de un trabajo precario respecto a salario, horas laboradas y prestaciones. Esta indagación implicó la realización de 30 entrevistas semiestructuradas, cuyos resultados permitieron validar las dimensiones de análisis mencionadas.

El objetivo de esta investigación es analizar en qué medida existe precarización del trabajo a partir del salario, jornada laboral y prestaciones sociales en el segmento de las mujeres en contraparte con los operadores hombres, a fin de establecer sí son las mujeres quienes laboran en condiciones de mayor trabajo precario, como establece la teoría de la temática.

Así que se justifica la investigación para identificar si en el mercado laboral mexicano existe el empleo precario y la existencia de diferencias entre los segmentos de hombres y mujeres a partir de las características establecidas al respecto por Mora (2012). De las variables establecidas por el autor, se retoman las siguientes: salario (hasta el ingreso de dos salarios mínimos es considerado precario), jornada laboral (baja jornada laboral si es de 15 horas y precario si es mayor a 48 horas) y prestaciones (si cuenta con las prestaciones de seguridad social, vacaciones, aguinaldo y derecho a crédito para vivienda o bien si no cuenta es considerado trabajo precario), así como si tiene representación sindical (sí la tiene o no. El carecer de representatividad sindical es parte del trabajo precario). 
Se parte de la siguiente hipótesis de trabajo: en el call center de Coppel, ubicado en el edificio La Lonja, se labora en condiciones de precarización del empleo, pero los trabajadores la aceptan por ser mujeres, jóvenes sin calificación y madres solteras, por la construcción social que hicieron de sí mismas sobre su origen socioeconómico; los empleos que realizaron previamente; y su condición de desempleados antes de llegar al centro de atención telefónica.

\section{Resultados de la entrevista: análisis cualitativos}

Durante la observación realizada, se detectó que el corporativo Coppel (tienda de ropa, muebles, electrodomésticos y servicios financieros con BanCoppel) cuenta en la ciudad de Culiacán con el servicio de centro de atención telefónica para México y América Latina. La forma en que se divide el personal es la siguiente:

- Edificio Margarita: 1.800 empleados (las llamadas se enfocan a cobranza)

- Lonja: 200 empleados (enfocados solo a promociones del corporativo Coppel y de Bancoppel). Fue el objeto de estudio.

- Andrade: 2.000 (cobranza)

- Obregón: 2.000 (atención a clientes y solicitud de crédito en línea)

Se trabaja dos turnos para México:

- Matutino: de 6:30am de la mañana a 1:40pm

- Vespertino: de 1:50pm a 8:50pm

1 turno para América Latina (Argentina y Brasil)

- Nocturno: entran a las 5pm y salen a las 4 am por el cambio de horario (trabajan en el edificio Obregón, el cual permanece abierto 24 horas)

Se les capacita intensamente, sobre todo para las llamadas internacionales. Incluso labora personal de origen brasileño, pero en nivel de jefe de turno.

De las entrevistas realizadas, los entrevistados coinciden en asegurar ser empleados de Coppel y en sus gafetes aparece el corporativo, pero otros empleados explican que son diferentes empresas al interior del grupo Coppel y la empresa que paga más y da prestaciones es el corporativo Coppel. Hay cierta ambigüedad en este punto.

Sobre los contratos laborales de los trabajadores del centro de atención telefónica, el 90\% de los entrevistados no supieron responder si eran empleados del corporativo Coppel directamente o subcontratados. 
Tienen las prestaciones mínimas establecidas en la Ley Federal del Trabajo: cuentan con seguro social a través del IMSS, una quincena de aguinaldo y trabajan en promedio siete horas diarias, pero en los días de fin de mes, cuando se acumulan más llamadas, pueden quedarse hasta nueve horas. Los trabajadores perciben un salario de 3 mil pesos quincenales, lo que es alrededor de 188 pesos diarios. Este aspecto ellos lo perciben como que gozan de grandes prestaciones y que su trabajo no es precario. Sin embargo, de acuerdo a las variables que califican el trabajo como precario (Mora, 2012), existen elementos para considerar que sí existe esta condición laboral, debido a que en términos de jornada laboral hay ocasiones en que laboran hasta nueve horas, realizan de 300 a 320 llamadas diarias con un intervalo de cinco minutos para ir al baño, carecen de comedor y tienen que comer en la calle o una plazuela aledaña. Esto significa en términos de jornada laboral más de 48 horas semanales, lo que sí configura el trabajo como precario.

En ese sentido, es importante reflexionar que implica un trabajo exhaustivo estar ante un conmutador telefónico en un espacio pequeño de 7 a 9 horas (sin poder levantarte de tu lugar), realizando de 300 a 320 llamadas telefónicas y solo poder ir al baño en una ocasión durante toda la jornada laboral.

En la segunda variable de seguridad social, cuentan con las garantías mínimas de ley, pero según narran no siempre les respetan el periodo vacacional de una semana por año laborado, ya que el jefe está en libertad de llamarlos en sus descansos sí se acumula el trabajo y es necesario acudir al centro de atención telefónica, para preservar su trabajo, pues existen dos casos que no acudieron y fueron despedidos. "Nos venden la idea de que nos van a canjear los días para después, cuando los ocupemos, pero eso no es verdad”, expresa Mario, uno de los empleados con dos años de antigüedad.

En la tercera variable relacionada con los salarios, que se consideran precario sí perciben hasta dos salarios mínimos, sí cumplen con esta característica, puesto que ganan en promedio 188.00 pesos diarios y no perciben ningún bono para transporte, despensa, ni cuentan con comedor de la empresa. Eso implica que de los 188.00 pesos que perciben diario gastan 50 pesos en alimento y 40 en camiones urbanos, dado que vienen de colonias periféricas de la ciudad y requieren cuatro camiones diarios para trasladarse a su lugar de trabajo. El salario que ganan alcanza apenas los dos salarios mínimos.

En el horario matutino laboran en su mayoría estudiantes universitarios de 19 a 22 años, así como mujeres. Existe en este turno baja rotación de personal. Los jóvenes los visualizan como un empleo temporal mientras acaban la universidad. Las mujeres lo ven como un trabajo de media jornada que les permite estar con sus hijos y las madres solteras como un gran apoyo económico, que les permite conciliar el mundo del trabajo y el mundo de la familia, cumpliendo así los múltiples roles que esto significa. 
También señalan que realizan en un día fuerte de 300 a 320 llamadas a Sinaloa, México, Argentina y Brasil. Por el cambio de horario, los de la noche son los que hacen las llamadas internacionales. En un día flojo son de 120 a 150 llamadas. Pasa un intervalo de 2 a 20 segundos para cada llamada. En los días flojos, los ejecutivos los ponen a leer sobre la filosofía gerencial de Coppel: misión, visión y valores. Tienen como jefes de turno a personal brasileño, pero eso no significa un conflicto intercultural, ya que señalan que son muy amigables y hablan bien español.

Se dicen muy contentos, pues tienen prestaciones como IMSS, una quincena de aguinaldo y trabajan en promedio siete a nueve horas diarias. Se califican a sí mismos como "bendecidos" por contar con estas prestaciones, pues la mayoría son estudiantes universitarias, mujeres (madres de familia y esposas) que por su doble rol solo pueden trabajar medio día. Lo que consideran la mayor bondad es que trabajan de siete a nueve horas diarias, que el resto del personal del turno de la mañana son jóvenes, realizan pausas activas de ejercicios aeróbicos cada tres horas, cuando los ejecutivos y gerentes así lo deciden. El 60\% del personal son mujeres.

Lo consideran como un empleo temporal, algo en lo que no se quedarían por toda la vida, ya que la mayoría actualmente estudia la universidad y en el caso de las jefas de familia dicen que es mientras sus hijos van a la escuela y logran graduarse. En un primer momento, niegan que sea un trabajo precario, pero después de contrastar sus respuestas encontramos elementos para considerar que sí hay precariedad laboral.

Quizás su construcción de la realidad o la forma en que le dan sentido a su trabajo, se deba a que la mayoría viene de colonias marginadas, en las cuales hacen una hora de distancia del centro de la ciudad; por lo que prefieren quedarse a desayunar o comer ahí, debido a que solo tiene 30 minutos para comer.

Anthony*4, un ejecutivo con tres años de antigüedad en la empresa y estudiante de segundo año de contabilidad revela que:

Este empleo es bueno, considerando que somos estudiantes y que no estamos sudando; estamos en la sombra y sentados. Que son muchas llamadas; a lo mejor sí, pero no es un empleo en donde nos quedaremos toda la vida. Es por mientras que acabamos la carrera (Anthony, comunicación personal, 30 de julio de 2018).

\footnotetext{
4 *Todos los nombres de los entrevistados fueron cambiados por cuestiones de ética científica.
} 
Mary*, colaboradora de 19 años, con seis meses de antigüedad y estudiante de primer año de periodismo dice:

Está muy bien, gano 3 mil a la quincena y nada más hago llamadas sobre las promociones de la tienda y algunas veces de servicios financieros. Me queda tiempo para ir a la universidad y estudiar lo que me gusta; pero, no pienso quedarme aquí toda la vida (Mary, comunicación personal, 28 de julio de 2018).

Mary* antes de llegar a ese empleo trabajó como mesera en una cadena nacional de restaurantes "María Antojitos" y como animadora de fiestas infantiles en McDonalds Culiacán, Sinaloa y dice:

En esos empleos ganaba mucho menos, eran de más de 8 horas diarias y no tenía las prestaciones que tengo ahora (Mary, comunicación personal, 18 de julio de 2018).

Francisca* de 43 años, con secundaria terminada, colaboradora con dos años en la empresa y madre soltera de dos niños dice en la entrevista:

Aquí está muy bien, pues ahora tengo mi quincena segura de 3 mil pesos, que no es mucho dinero, pero me permite mantener a mis hijos y tener un trabajo seguro. Antes no tenía empleo y batallaba muchísimo para mantenerlos. Ahora tengo hasta seguro social, aguinaldo y no más trabajo siete horas. Los últimos días del mes hay muchas llamadas, pero uno le va agarrando el modo y pues ahí vamos (Francisca, comunicación personal, 25 de julio de 2018).

No obstante que, en los tres casos, existen condiciones para definir el trabajo del centro de atención telefónica como precario: los empleados tienen una perspectiva subjetiva y lo ven como un trabajo decente. Es decir, tienen su propia construcción social de su realidad laboral, la cual se da en función de tres factores:

1. Su origen socioeconómico

2. Los empleos que realizaron previamente

3. Su condición de desempleados antes de llegar al centro de atención telefónica

Al analizarlas variables de trabajo precario en las dimensiones de jornada laboral, salario y prestaciones en los trabajadores, objeto de estudio se encontró que cumplen con las características de un trabajo precario en términos de salario y jornada laboral, pues ganan 
apenas 3 mil pesos a la quincena e invierten 750 pesos en camiones para trasladarse de su casa al centro de trabajo y otros 750 en alimentos, ya que carecen de comedor. Es decir, que en términos absolutos les quedan 750.00 pesos por quincena para sus necesidades de vivienda, alimentos, educación, esparcimiento, etc., cuya cantidad es insuficiente para tener una vida digna, sobre todo para quienes tienen dependientes económicos.

Sin embargo, una característica de este grupo estudiado es que se muestran optimistas ante el futuro laboral, aunque admiten que no se quedarán aquí toda la vida, precisamente por las condiciones laborales descritas. Para Juana* de 55 años, es un trabajo de apoyo a su marido, mientras logra un mejor empleo:

Voy a estar aquí de seis meses a un año y cuando mi esposo logre colocarse en alguna chamba, pues dejo de trabajar. Es por mientras; por eso no me quejo de las 300 llamadas. Mejor aquí que como criada en alguna casa de ricos", dice, mientras sonríe (Juana, comunicación personal, 23 de julio de 2018).

Las mujeres que solo tienen estudios de secundaria y que son mayores de 50 años, revelan que por su baja escolaridad y por pasar de esa edad difícilmente podrían colocarse en el mercado de trabajo. En el caso mexicano, tener medio siglo de edad representa una barrera cultural para acceder a un nuevo empleo (pues solo le quedan 15 años de vida productiva, para acceder a la pensión en términos de legislación laboral). Ante ello, la entrevistada añade:

Las condiciones de esta chamba no son las mejores, pero como una ya está entrada en años y no fue muchos años a la escuela; no queda más que apechugar lo que digan los patrones. Se necesita mantener a la familia (Juana, comunicación personal, 20 de julio de 2018).

En tanto, José* un exgerente del área de sistemas revela:

Te venden la idea de que eres empleado de Coppel y que vas a ir creciendo en la empresa, que hay muchas oportunidades de desarrollo, pero eso no es cierto. Para cambiarte de un puesto a otro tienen que pasar a veces años; claro que como en todos lados depende de con quién te juntes. Como en todo en México hay que tener palancas. Yo sí supe con quién juntarme y a los seis meses estaba en gerencia de programación. Lo malo de las promociones es que el proceso de cambio dura seis meses y hasta después de tres meses en tu nuevo puesto te dan el nuevo sueldo, lo cual significa que trabajas nueve meses con el salario de colaborador y en realidad hacías trabajo de gerencia, como a mí me paso (...). Te venden mucho la idea de la filosofía gerencial, de la escuela de Coppel, pero la realidad es que 
quienes se quedan son los que se conforman con poco por su condición de mujeres, estudiantes o con baja escolaridad (José, comunicación personal, 02 de agosto de 2018).

Sin embargo, reconoce que es positivo para estudiantes, madres solteras y personas con discapacidad, que en otras condiciones difícilmente pudieran laborar, por razones de las construcciones sociales que existe sobre estas personas.

Con esa investigación se observó que los trabajadores tienen en el call center, las prestaciones, ingresos y jornadas laborales consideradas como mínimas en la Ley Federal del Trabajo Mexicana, lo que es un indicador bueno, pues no están bajo la informalidad laboral que es un eje de precariedad e inseguridad mayor para el trabajador. Sin embargo, dista mucho de ser considerado un trabajo decente en términos de la OIT. Pero es un trabajo muy exhaustivo, con más de 300 llamadas diarias, en las siete o nueve horas de trabajo. Los trabajadores mencionaban ese hecho, pero siempre buscando una justificación por la razón que lo aceptan.

Cabe mencionar que, en la parte de seguridad laboral, es fundamental que cuentan con un contrato laboral (Mora, 2012); sin embargo, los trabajadores analizados no sabían sí estaban contratados por la empresa multinacional Coppel o eran subcontratados. Esa situación refleja dos aspectos: primero, la falta de información y claridad sobre la empresa y las reglas bajo la cual están contratados los trabajadores, que si es factor de inseguridad y precariedad laboral; y segundo, por su baja escolaridad no entienden la importancia de contar con seguridad laboral.

Para esta investigación, quedó claro que este trabajo no exige calificación del trabajador, aunque según los entrevistados, la empresa los apoya a estudiar, con abonos o apoyando con $50 \%$ de los pagos de la escuela.

Otro aspecto a destacar es que coinciden en señalar que es un trabajo temporal y que no planean tener una trayectoria laboral dentro de la empresa. Ese hallazgo coincide con el planteamiento del trabajo líquido de Bauman (2000), quien establece que en la sociedad posmoderna los trabajadores cambian de trabajo en forma continua y forma parte de su búsqueda de identidad o realización personal. Ese fue la respuesta más importante de la investigación, pues los trabajadores no se sentían precarizados, mejor dicho, se sentían en un trabajo decente, pero sin ganas o idea de hacerlo por toda la vida. Así que, se observa que en la verdad no lo tiene como un buen trabajo, seguro u objeto de grandes avances profesionales, lo que se concluye por un trabajo bajo condiciones precarias en términos de salario y jornada laboral. 
En general, se puede concluir que la cuestión del trabajo precario, con indicadores macrosociales y estadísticos de análisis sí son las mujeres las más precarizadas en el caso mexicano (ENOE: INEGI, 2005-2018), lo cual comprueba parcialmente la hipótesis y ofrece una mirada general de la realidad. Pero, en el nivel micro social (analizado a partir del estudio de caso) se encontró que no existen diferencias sustanciales en las condiciones laborales de los hombres y mujeres en el centro de atención telefónica; es decir, que están igualmente precarizados. Sin embargo, las mujeres entrevistadas se quejaban de que realizan una doble jornada, pues al llegar a su casa deben asumir el rol de esposas, madres de familia y amas de casa, lo cual significa que laboran dentro del hogar ocho horas diarias en promedio.

Esto significa que no obstante la situación de posmodernidad en el mundo del trabajo, en el ámbito de la familia las mujeres se siguen sintiendo como las únicas responsables de familia, por las tareas domésticas y el cuidado de los enfermos, lo cual las sitúa en una condición de desventaja en el mundo laboral.

Al final, la hipótesis planteada se ha comprobado parcialmente, dado que en este centro de trabajo tanto hombres como mujeres están en condiciones igualmente precarias y existen diferentes racionalidades para aceptar esas condiciones laborales. En el caso de las mujeres, lo aceptan por ser mujeres, sin calificación laboral y madres solteras, así como por la construcción social que realizan de sí mismas por su origen socioeconómico, los empleos que realizaban previamente y su condición de desempleo que enfrentaban antes de llegar al call center. En el caso de los hombres, aceptan este trabajo precario por ser jóvenes, estudiantes y por visualizarlo como un trabajo temporal. Un hallazgo interesante de esta investigación es que un nuevo segmento precarizado en el mundo del trabajo son los jóvenes, sobre todo en México, en donde 3,6 millones de jóvenes no estudian ni trabajan (OCDE, 2016) y esa cifra representa el $22 \%$ de la población.

\section{Conclusiones}

Al final en este trabajo se ha alcanzado a identificar los cambios en el mercado laboral contemporáneo globalizado en México, en donde la sociedad posmoderna implica un ingreso cada vez mayor de las mujeres como fuerza de trabajo, aunque las condiciones de trabajo no sean siempre las mejores, de acuerdo al análisis macrosocial.

Sin embargo, en el estudio microsocial emerge otro grupo segmento de la población como precarizado que son los jóvenes, quienes de acuerdo con Bauman (2010), experimentan el mundo del trabajo como algo temporal y efímero. 
Los jóvenes representan una fuerza laboral muy importante, que acepta trabajos precarios bajo el argumento de que quieren un trabajo de tiempo parcial, son estudiantes y lo que buscan son experiencias, pero que no se quedarán en ese empleo más allá de lo que duren sus estudios universitarios o que decidan irse a laborar a otro estado o país. Es decir, visualizan las trayectorias laborales en forma distinta y no es la estabilidad laboral un aspecto que se encuentre en el centro de sus preocupaciones, lo cual confirma la tesis de Bauman del trabajo líquido en las nuevas generaciones.

Con esta investigación se cuestiona el mito de que son las mujeres el grupo sociocultural que se enfrenta a una situación laboral de mayor precariedad, sino que tanto hombres como mujeres experimentan el trabajo precario en similares condiciones. Este hallazgo es aún más revelador, pues el lograr el trabajo decente en términos de los establecido por la Organización Internacional del Trabajo (OIT) se torna en un desafío mayor tanto para los trabajadores, como para los gobiernos que deberán generar políticas públicas y legislaciones laborales que pugnen cada vez más por alcanzar este objetivo.

El objetivo central de la investigación fue identificar en qué medida existe precarización del trabajo para las mujeres en el mercado laboral mexicano. Los resultados han sido que en el análisis general de la ENOE 2005-2018, existen más mujeres en la población total de México, pero son hombres los que tienen más oportunidad de un empleo subordinado y remunerado, es decir, formal, con contrato de trabajo que les da prestaciones, vacaciones, aguinaldo y seguro social. También son las mujeres que reciben los ingresos más bajos, bajo un salario mínimo, aún también son las que tiene una jornada laboral, más larga, de 48 horas semanales.

Sin embargo, en el estudio microsocial la hipótesis no fue comprobada, lo cual significa que hombres y mujeres enfrentan el trabajo precario, aunque existen distintas racionalidades (por cada género y grupo de edad), para aceptarlo. Las mujeres aceptan el trabajo precario por considerar que es de medio tiempo, que les permitirá cumplir con la doble jornada y ser madres de familia y esposas, tal como lo establece la tradición cultural en México. En el caso de los hombres lo aceptan por ser jóvenes, estudiantes y por ser una alternativa momentánea para pagar sus estudios, así como una experiencia de vida.

Esta investigación ha buscado responder la cuestión de la precarización del trabajo en el contexto social globalizado, identificando retos laborales hacia a un trabajo decente para hombres y mujeres, incluso con la finalidad de contribuir al desarrollo de políticas públicas para disminución de la brecha de género, pero también para que se ofrezcan mejores condiciones laborales a los jóvenes y puedan aspirar ambos grupos socioculturales a un trabajo decente. 
Las líneas de investigación futura que se derivan de este trabajo es analizar la situación de trabajo precario en los jóvenes, así como la racionalidad con la que aceptan estas condiciones laborales, propias de la sociedad posmoderna. Otra línea que valdría la pena explorar es la nueva visión que existe en cuanto a la estabilidad laboral, dado que el grupo de trabajadores analizados no la considera como un elemento relevante. En la sociedad moderna la estabilidad laboral era fundamental y el mundo del trabajo, así como las trayectorias laborales se explicaban en función de este elemento; sin embargo, en la llamada sociedad posmoderna, en el mundo del trabajo emergen nuevos elementos que vale la pena analizar y reflexionar en trabajos futuros.

\section{Referencias bibliográficas}

Abramo, L. (2006). Trabajo decente y equidad de género en América Latina. Ginebra, Suiza: Oficina Internacional del Trabajo.

Anker, R., Chernyshev, I., Egger, P., Mehran, F., y Ritter, J. (2003). La medición del trabajo decente con indicadores estadísticos. Revista Internacional del trabajo, 122(2), 161-195.

Antunes, R. (2000). Las metamorfosis en el mundo del trabajo. Nómadas (Col), (12), 28-37.

Antunes, R. (2009). O Trabalho, Sua Nova Morfologia e a Era da Precarizacao Estrutural. Theomai: estudios sobre sociedad, naturaleza y desarrollo, (19), 47-57.

Antunes, R. (2015). O caracol e sua concha: ensaios sobre a nova morfologia do trabalho. Sao paulo, Brasil: Boitempo Editorial.

Antunes, R., y Braga, R. (2009). Infoproletários: degradação real do trabalho virtual. São Paulo, Brasil; Boitempo Editorial.

Bauman, Z. (2000). Trabajo, consumismo y nuevos pobres. Barcelona, España: Editorial Gedisa.

Bauman, Z. (2010). Modernidade líquida. Rio de Janeiro, Brasil: Editora Zahar.
Bauman, Z., y Bordoni, C. (2016). Estado de crisis. Barcelona, España: Paidós.

Beck, U. (2000). Un nuevo mundo feliz: la precariedad del trabajo en la era de la globalización. Barcelona, España: Paidós.

Braga, R. (2012). A Política do precariado: do populismos à hegemonia lulista. São Paulo, Brasil: Boitempo.

Gálvez, E., Gutiérrez, E., y Picazzo, E. (2011). El trabajo decente: nuevo paradigma para el fortalecimiento de los derechos sociales. Revista mexicana de sociología, 73(1), 73104. Recuperado de http://www.scielo.org.mx/scielo.php? script=sci_arttext\&pid=S0188 $25032011000100003 \& \operatorname{lng}=$ es\&tlng= es

García, G., y Perez, C. (2007). La precarización del empleo en México, 1995-2004. Revista Latinoamericana de Estudios del Trabajo (RELET), 12(19), 39-78.

Ghai, D. (2003). Trabajo decente. Concepto e indicadores. Revista internacional del trabajo, 122(2), 125-160.

Guzmán, B. (2009). Mexican Urban Labor Markets in the Early 21st Century. 
Revista mexicana de sociología, 71(1), 5-46.

Hirata, H. (2000). Relaciones sociales de sexo y división del trabajo. Contribución a la discusión sobre el concepto de trabajo. Herramienta. Recuperado de http://fundacionjyg.org/wpcontent/uploads/2018/09/Relacionessociales-de-sexo-y-divisio\%CC\%81ndel- trabajo-Contribucio\%CC\%81n-a-ladiscusio\%CC\%81n-sobre-el-conceptotrabajo.pdf

Hirata, H. (2002). Globalização e divisão sexual do trabalho. Cadernos Pagu, (17-18), 139-156. Recuperado de https://dx.doi.org/10.1590/S010483332002000100006

Horbath, J., y Gracia, A. (2014). Discriminación laboral y vulnerabilidad de las mujeres frente a la crisis mundial en México. Economía, sociedad y territorio, 14(45), 465-495. Recuperado de http://dx.doi.org/10.22136/est002014 382

Instituto Nacional de Geografía y Estadística (INEGI). (2005-2018). Encuesta Nacional de Ocupación y Empleo (ENOE). Ciudad de México. Recuperado de www.inegi.org.

Kalleberg, A. (2009). O crescimento do trabalho precário: um desafio global. Revista Brasileira de Ciências Sociais, 24(69), 21-30.

Kalleberg, A. (2011). Good Jobs, Bad Jobs: The Rise of Polarized and Precarious Employment Systems in the United States, 1970s-2000s. Nueva York, Estados Unidos: Russell Sage Foundation.

Kergoat, D. (2003). De la relación social de sexo al sujeto sexuado. Revista mexicana de sociología, 65(4), 841-
861. Recuperado de http://www.scielo.org.mx/scielo.php? script=sci_arttext\&pid=S018825032003000400005\&lng=es\&tlng= es.

Marx, K. (1985). O Capital: crítica da economia política. São Paulo, Brasil: Expressão Popular.

Marx, K. (2010). Trabalho assalariado $e$ capital \& Salário, preço e lucro. São Paulo, Brasil: Expressão Popular.

May, T. (2004). Pesquisa social. Porto Alegre, Brasil: Artmed.

Mora, M. (2012). La medición de la precariedad laboral: problemas metodológicos y alternativas de solución. Trabajo, 5(9), 87-122.

Navarro, G., y Gámez, R. (2006). Mujeres: factor de cambio: ejecutivas $y$ empresarias sinaloenses. Culiacán, México: Universidad Autónoma de Sinaloa.

OIT. (2017). Informe mundial sobre os Salários 2016/17. Recuperado de https://www.ilo.org/wcmsp5/groups/ public/---dgreports/---dcomm/--publ/documents/publication/wcms_5 41632.pdf

Pérez, J. (2016). Derechos laborales: una mirada al derecho a la calidad de vida en el trabajo. CIENCIA ergo-sum, Revista Científica Multidisciplinaria de Prospectiva, 23(2), 121-133.

Ridgeway, C. (1992). Gender, interaction, and inequality. Nueva York, Estados Unidos: Springer.

Ridgeway, C. (2011). Framed by Gender: How Gender Inequality Persists in the Modern World. Oxford, Reino Unido: Oxford University Press.

Ridgeway, C. (2014). Why Status Matters for Inequality. American Sociological 
Review, 79(1),1-16.

doi: 10.1177/0003122413515997

Santos, B de S. (1993). O Estado, as relações salariais e o bem-estar social na semiperiferia: o caso português. En: Boaventura de Sousa Santos (Org.). Portugal: um retrato singular (pp.1756). Porto: Edições Afrontamento.

Sassen, S. (2010). A Sociology of Globalization. Londres, Reino Unido: Routleged.

Sen, A. (2000). Trabajo y derechos. Revista internacional del trabajo, 119(2), 129-139.

Standing, G. (2014a). O Precariado: A nova classe perigosa. 1. Belo Horizonte, Brasil: Autêntica Editora.

Standing, G. (2014b). O precariado e a luta de classes. Revista Crítica de Ciências Sociais, (103), 09-24. Recuperado de https://dx.doi.org/10.4000/rccs.5521

Stiglitz, J. (2002). Empleo, justicia social y bienestar de la sociedad. Revista internacional del trabajo, 121(1-2), 9-31.

Tilly, C., y Tilly, C. (1998). Durable inequality. Berkeley, Estados Unidos: University of California Press.

Valencia, A. (1999). Globalización y precarización del trabajo en México. México. Ediciones El Caballito.

Valencia, A. (2015). El precariado: ¿nueva clase social? Universidad Nacional Autónoma de México, Facultad de Ciencias Políticas y Sociales, Posgrado en Estudios Latinoamericanos. 